\title{
THEMATIC PAPER \\ Reorienting health systems in South-East Asia to deliver care for mental and neurological disorders
}

\author{
Nazneen Anwar, ${ }^{1}$ Vijay Chandra ${ }^{2}$ and Athula Kahandaliyanage ${ }^{3}$
}

Temporary International Professional, Mental Health and Substance Abuse, WHO SEARO

${ }^{2}$ Regional Advisor, Mental Health and Substance Abuse, WHO SEARO, email chandrav@searo. who.int

${ }^{3}$ Director, Department of Sustainable Development and Healthy Environments, WHO

The authors are staff members of the World Health Organization. The authors alone are responsible for the views expres in this publication they do not necessarily represent the decisions, policy or views of the World Health Organization.

The authors gratefully acknowledge the contributions made by the following in Bangladesh, National Institute of Mental Health:

Dr Waziul Alam Chowdhury

Dr Helal Uddin Ahmed

Dr Faruq Alam

Dr Abdul Mohit

Bhutan:

Mr Tandin Chogye

Dasho Kuenzang N. Tshering

Mrs Zangmo

Timor-Leste:

Dr Gaspar Quintao

Mr Teofilio Julio Kehie Tilman

Mr Jacinto Vila Nova implementing the epilepsy project at the field level.

Traditionally, care for mental and neurological disorders has been concentrated in tertiary care hospitals located in large cities. These custodial types of facility were designed to 'protect' the community from patients with a mental illness, as such persons were considered dangerous and $a$ threat to the community. Given the state of medical knowledge in the 19th and 20th centuries, this mode of care was considered appropriate. However, in recent decades more humane and effective concepts of care have evolved. These concepts recognise the stigma attached to hospital-based care and also its limited outreach to the community, leaving out the vast majority of people living in rural and remote areas. Violation of human rights, sometimes seen in mental hospitals, has also been of concern.

The burden of mental and neurological disorders is staggering. According to the World Health Organization (WHO), approximately 450 million people globally suffer from mental, neurological, behavioural or substance use disorders. These disorders account for $13 \%$ of the global burden of disease and significantly contribute to comorbidity in other diseases (WHO, 2008). Until recently, however, little importance was given to the morbidity caused by these disorders, as they did not appear in 'cause of death' statistics on which public health priorities were based. Accordingly, the WHO has developed the concepts of disabilityadjusted life-years and years lived with disability. Using these measures as an indicator of suffering,

\section{Table 1}

Leading causes of years lived with disability (YLD) in low- and middle-income countries, 2004

\begin{tabular}{|c|l|l|l|}
\hline Rank & Disability & YLD (millions) & $\%$ of total YLD \\
\hline 1 & Unipolar depressive disorders & 55.3 & 10.4 \\
\hline 2 & Refractive errors & 25.0 & 4.7 \\
\hline 3 & Hearing loss, adult onset & 23.2 & 4.4 \\
\hline 4 & Alcohol use disorders & 18.4 & 3.5 \\
\hline 5 & Cataracts & 17.4 & 3.3 \\
\hline 6 & Schizophrenia & 14.8 & 2.8 \\
\hline 7 & Birth asphyxia and birth trauma & 12.9 & 2.4 \\
\hline 8 & Bipolar disorder & 12.9 & 2.4 \\
\hline 9 & Osteoarthritis & 12.8 & 2.4 \\
\hline 10 & Iron-deficiency anaemia & 12.6 & 2.4 \\
\hline
\end{tabular}

Source: WHO (2008) mental and neurological disorders are high on the list of causes of morbidity (Table 1).

To address this huge burden of mental and neurological disorders, there is a great scarcity of trained professionals and infrastructure in member states of the WHO South-East Asia Region (SEAR). According to the Mental Health Atlas 2011, the median number of psychiatrists globally is 1.27 per 100000 population, whereas in SEAR the corresponding figure is 0.23 (WHO, 2011). The global number of psychologists is 0.3 per 100000 , compared with 0.03 in SEAR. Globally, while there are 0.23 and 0.05 social workers and occupational therapists per 100000 population respectively, the numbers in SEAR are negligible. Mental health expenditure per capita is 200 times greater in high-income countries compared with low-income countries, such as the countries in SEAR (WHO, 2011).

Evidence of the limited effectiveness of the traditional hospital-based care for mental and neurological disorders is the large 'treatment gap' in the community. The treatment gap is the difference between the number of people with a disease and the number of people being appropriately treated in a given population at a given point in time, expressed as a percentage. The treatment gap has been documented in SEAR member states to be between $76 \%$ and $85 \%$ for epilepsy and psychosis. For high-income countries the estimates range from $35 \%$ to $50 \%$ (WHO Regional Office for South-East Asia, 2012). Such large numbers of people being deprived of treatment are unacceptable in light of commendable advances made in medical science, including better understanding of diseases, better treatment, better availability of effective psychotropic medication and better management

Recognising the reality of the existing situation in the community - that is, the huge burden, large treatment gap, limited infrastructure and workforce, and the substantial rural and remote areas to be served - the WHO's Regional Office for South-East Asia (SEARO), in collaboration with regional experts, has developed some innovative approaches. The SEARO strategy is to treat and manage patients in the community by empowering existing national primary healthcare systems. The strategy calls for training of community-based health workers in the identification of priority mental and neurological disorders and then treatment by a primary care physician. 
However, it is unrealistic to expect the primary healthcare system to deliver comprehensive care for all types of neuropsychiatric conditions. The SEARO strategy addresses only the most common, most disabling conditions, easy to identify through observation, the availability of low-cost medication and good prognosis with treatment. Accordingly, epilepsy, psychosis and depression are the three conditions that have been identified in most of the member states as priority mental and neurological disorders for intervention.

In developing services through the existing primary healthcare system, SEARO has taken a 'five A' approach: Availability, Acceptability, Accessibility, Affordable medications and Assessment.

- Availability. Services that will address at least the minimum needs of populations in relation to mental and neurological disorders should be available to everyone regardless of where they live.

- Acceptability. Populations need to be informed and educated about the nature of neuropsychiatric illnesses, so that they accept these services.

- Accessibility. Services should be available to the community, in the community and at convenient times.

- Affordable medications. Every effort should be made to guarantee uninterrupted supplies of essential medications at reasonable cost.

- Assessment. These programmes need to be continually assessed to ensure their appropriateness and cost-effectiveness.

SEAR member states have excellent existing primary healthcare systems, which reach out to all areas of the country, including rural and remote areas. Bangladesh, for instance, has 11500 community clinics, each of which caters for a population of around 6000 in rural areas. The next tier of care is the upazila health complex, where communitybased doctors provide services to patients referred from the community clinics. In Bhutan primary healthcare services are provided through the basic health units located in villages. The district hospitals in Bhutan run community health programmes through 518 outreach clinics. These clinics give referral support to the basic health units. In Myanmar the primary healthcare system reaches out to the community. A well established referral system exists from the village to the township level and to the tertiary care hospital. In Timor-Leste primary healthcare services are provided through a network of district hospitals and community health centres. Facility-based services are provided to the community through a network of health posts. In Thailand there are two health volunteers in each village who serve as the first level of contact leading to a well developed primary, secondary and tertiary referral health service.

To pilot this strategy, SEARO has supported epilepsy projects in Bangladesh, Bhutan, Myanmar
Table 2

Epilepsy treatment gap ( \% ) before and after intervention

\begin{tabular}{|l|l|c|}
\hline Country & Before intervention & After intervention \\
\hline Bangladesh & 87.0 & 5.0 \\
\hline Bhutan & 40.0 & 26.0 \\
\hline Myanmar & 94.6 & 5.0 \\
\hline Timor-Leste & 70.7 & 53.7 \\
\hline
\end{tabular}

and Timor-Leste. Under this project communitylevel health workers were trained to identify and refer individuals with epilepsy to primary care physicians. An easy-to-use identification tool, developed by experts from SEAR, was used for the identification of the probable cases in the community. Training manuals were translated into the local language for convenience of the health workers. A continuous supply of medicine (phenobarbitone) was ensured.

An impact evaluation of each project was conducted, taking percentage reduction in the treatment gap for epilepsy as an indicator. On evaluation of the projects, a substantial reduction in the treatment gap for epilepsy was seen (Table 2). This proved that it was possible to deliver care for people with epilepsy efficiently through the existing primary healthcare system.

In conclusion, the findings of the pilot projects conducted in Bangladesh, Bhutan, Myanmar and Timor-Leste have been encouraging. They provide evidence that even in low-resource countries it is possible to deliver care for persons with mental and neurological disorders by empowering the existing primary healthcare system. This innovative service model is sustainable in the long term since it can be mainstreamed into the existing government healthcare system with minimal additional investment in infrastructure. It reaches out to all those in need of care, provides care to people in the community and is less stigmatising. Similar strategies will be developed for the identification and management of psychosis and depression.

\section{References}

WHO (2008) Global Burden of Disease 2004 Update. World Health Organization.

WHO (2011) Project Atlas: Resources For Mental Health. World Health Organization.

WHO Regional Office for South-East Asia (2012) Report of the Regional Meeting on Noncommunicable Diseases Including Mental Health and Neurological Disorders. WHO Regional Office for SouthEast Asia.

'He who has health has hope, and he who has hope has everything.' (Proverb) 